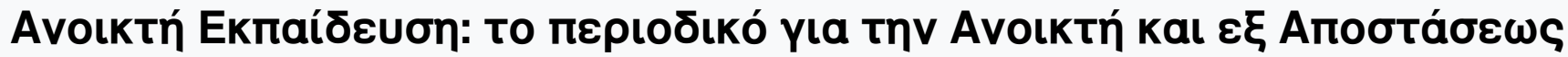

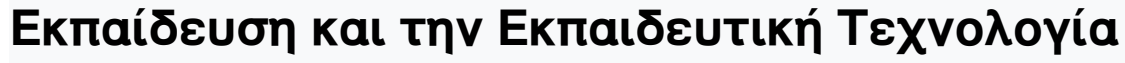

Tóp. 9, Ap. 1 (2013)

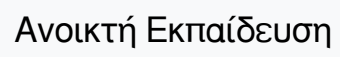

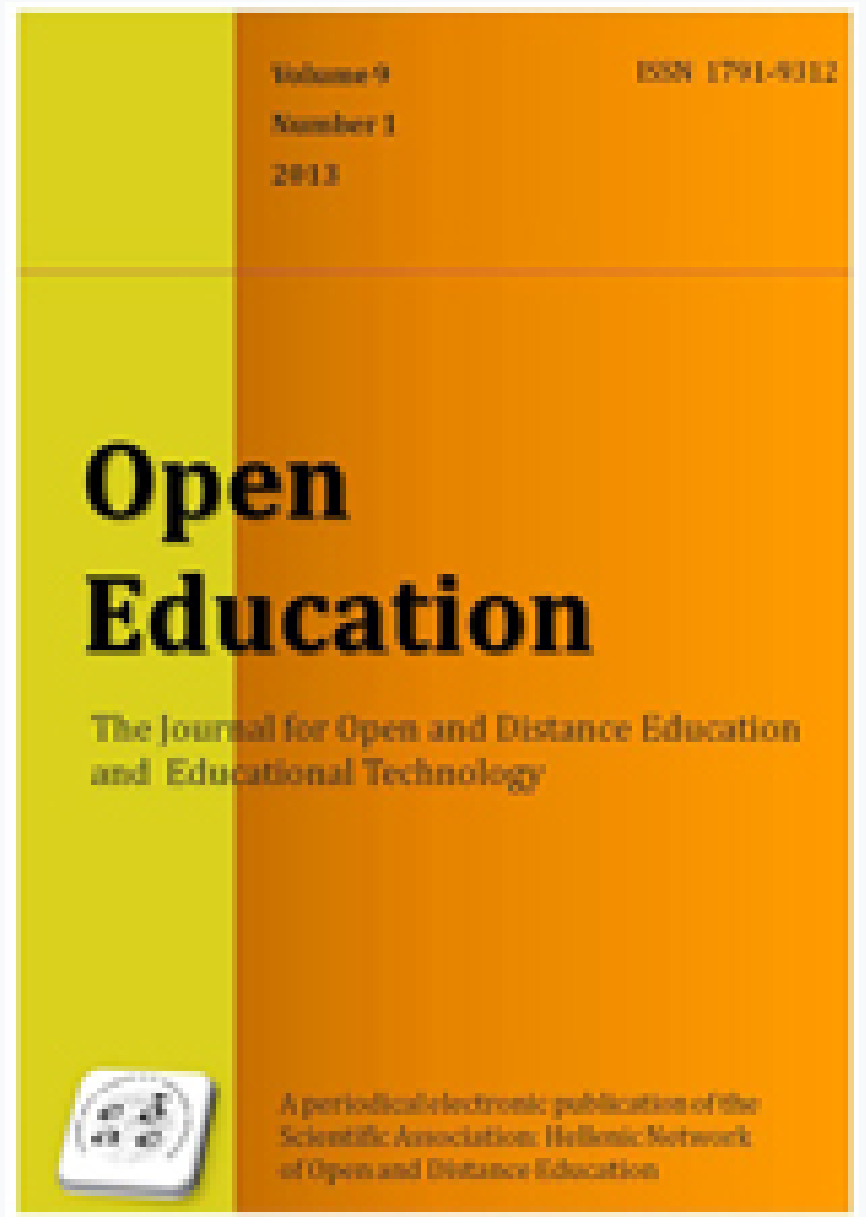

Teleconference in support of distance learning: Views of educators

Christos Panagiotakopoulos, T. Tsiatsos, Antonis

Lionarakis, Nikolaos Tzanakos

doi: $\underline{10.12681 / \text { jode. } 9806}$

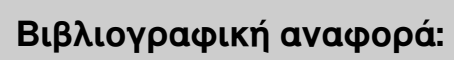




\title{
Teleconference in support of distance learning: Views of educators
}

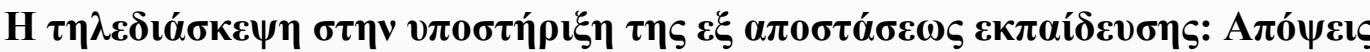 $\tau \omega \mathrm{v} \varepsilon \kappa \pi \alpha \iota \delta \varepsilon \tau \omega \dot{v}$}

Christos Panagiotakopoulos Department of Primary Education, University of Patras cpanag@upatras.gr

\section{T. Tsiatsos}

Department of Informatics, Aristotle University of Thessaloniki tsiatsos@csd.auth.gr

Antonis Lionarakis

Hellenic Open University alionar@otenet.gr

Nikolaos Tzanakos

Hellenic Telecommunications Organization (OTE) ntzanakos@ote.gr

\begin{abstract}
In this study we recorded the views of the educators that use teleconference as an educational medium to support distance learning. The data were collected using the semi-structured interview and the results showed that teleconferencing could be a strong complementary tool to support flexible learning as well as distance education, by enhancing the interaction among learners. All the interviewees consider teleconference as a "helpful assistant" in the educational process and suggested that the educators should be well prepared before the tele-lecture, as well as prepare and guide the learners in order to conduct a smooth teleconferencing session. From the interviews, several advantages and disadvantages of the teleconference's use as an educational tool emerged. The main advantages consist of the independence of space and time while conducting learning sessions, the easy access to and sharing of educational content, the interaction between educator and learners as well as among the learners and the reduced transportations along with time saving. On the other hand, some important disadvantages refer to the participants being unfamiliar with technology, lack of technological support, limited network resources causing disconnections and adding stress to participants, the uncertainty of the participants regarding the effectiveness of teleconference in education and the lack of face-to-face communication.
\end{abstract}

\section{Keywords}

\section{Teleconference, distance learning}

\section{Introduction}

Distance learning (DL), is an educational method, a teaching and learning methodology characterized by the distance that separates the educator from the learner. The term is used, in its primary form, to describe a learning process in which a significant part of the teaching is performed by the educator, who is removed from the learner in terms of location and time (Perraton, 1988). 
In a first consideration, if we take into account that education is essentially based on a relationship of dialogue and constant interaction (Simpson, 2000), DL compared to face-to-face learning is offered with one intrinsic factor acting negatively to dialogue development: the distance that separates educators from learners. This is the exact point that technology can help to attenuate the negative effects of this factor.

During the last decade, the rapid development of networks and telecommunications, as well as Information and Communication Technologies (ICTs), has helped to open up new horizons, new capabilities and new ways of interaction. In education, the use of technology can improve learning conditions by enhancing educator-learner interaction and/or learner - learner interaction (Panagiotakopoulos, Lionarakis \& Xenos, 2003), as well as collaboration in either synchronous or asynchronous learning environments (Beldarrain, 2006).

Towards this direction, a lot of research effort has been put on providing several technological solutions for the enhancement of interactivity and collaboration in DL, including teleconference (Roberts, 2009; Anastasiades, Filippousis, Karvunis, Siakas, Tomazinakis, Giza \& Mastoraki, 2010), virtual spaces (Baker, Wentz, and Woods, 2009; McBrien, Cheng \& Jones, 2009) and social networking tools and applications (Hoffman, 2009; Hatzipanagos \& Warburton, 2009). According to Tzanakos research (2012) and regardless of the technological approach, however, the educator's role must receive special attention concerning the design of the supporting material and human contact. Physical contact and face to face communication should not be replaced by the Internet or any other technological device.

Virtual or distance class has received enormous attention at a research level, facilitating social presence and fostering socialization among distance learners for collaborative learning activities (Edirisingha, Nie, Pluciennik \& Young, 2009). These computer-mediated virtual learning environments combine pedagogical, communication and administration software tools integrated into one system that can be used to promote learning (Ellaway, Dewhurst \& Cumming, 2003).

Usually, one of the main supporting levers of this type of classrooms is e-learning that refers to learning mediated by computers, electronic devices and the Internet as a means of connection and data transfer (Tzanakos, 2012).

According to a recent research conducted in Duke University with students enrolled in a distance-based graduated nursing informatics class, while learners show preference for synchronous web-based learning, virtual environments emerged as the favored distance based education among three different online modalities of learning (Johnson, Corazzini \& Shaw, 2011). However, sophisticated virtual environments, usually developed for gaming, recreation and entertainment, are little researched in terms of their educational uses (Edirisingha, Nie, Pluciennik \& Young, 2009).

In practice, one widely adopted technological tool to strengthen interaction and collaboration in a distance class is teleconference. The term teleconference is a compound word consisting of the Greek ancient adverb "Tele" (from far) and the noun "conference". Interpreting the term from its technological perspective, we would define it as "real time data exchange, between humans located at different physical spaces who are connected via a telecommunication system". Teleconference exploits the new capabilities offered by new and emerging ICTs, which guarantee transmission of different types of information and enable immediate, vivid and often 
bidirectional connection between the educator and the learner, who are in different locations and most of the times at a great distance (Tzanakos, 2012).

Thus, through teleconference, animation, audio and data can be transmitted to large spaces, where screens and sophisticated audiovisual media can be found. It can be realized through several means, such as Personal Computers (PCs), Personal Digital Assistants (PDAs), tablet PCs, stationary computer terminals and mobile phones. A key feature of teleconference in education is the ability to support DL using internet technology in real time (Rozi, 2007) creating virtual environments in their simplest form. This type of meeting in a virtual space maximizes learning efficiency by increasing the interaction between instructors and learners, as the distance gap is now "bridged". Educators and learners act in parallel and at the same time, even if they are not located at the same physical space. Nevertheless, it has to be mentioned that in DL, this technological tool is not intended to replace face-to-face teaching, but to complement it (Anastasiades, 2006).

Teleconference began with timid steps, compared with other Internet technologies, due to the limited data transfer rates that prevailed until recently in networks significantly reducing the amount of data they could handle. The continuous increase in network speeds gave a strong motivation for the scientific community to develop and implement a very effective communication and educational tool (Tzanakos, 2012). Nowadays, improved speeds in networks and especially the Internet facilitate the transmission of high resolution images enabling High definition Video Conferencing (HDVC). HDVC is an evolving technology of vital significance in the fields of medicine, entrepreneurship, education etc. (Trueb, Lammers \& Calyam, 2007). Today, there are plenty of available tools (software and hardware) facilitating teleconference (e.g. Adobe Connect, e:Presence, Big Blue Button, Sony, Tandberg, Polycom, LifeSize, Banckle Online Meeting, Blackboard Collaborate, Fastviewer, iLink, LiveOn, Centra, Saba Centra, RHUB, WizIQ, WebEx), which through constant upgrades have reached a point, where even the most demanding users in terms of quality and service can be satisfied.

While conducting an educational act with the use of teleconference, educators play an essential and dominant role. In this paper, we intend to elicit and highlight the positive and negative aspects of the teleconference-supported educational process based on the experience of educators using teleconference systems.

Highlighting the positive and negative aspects of using teleconference as an educational medium from the instructors' perspective, will provide insight into several issues. For example, it will give educators that implement it the opportunity to understand that teleconference is a challenge for them, not so much regarding the use of technological equipment, rather than in terms of operating capabilities, towards a more effective educational practice. Teleconference is a simple technological tool, but it requires awareness of what its use can and cannot offer from an educational point of view, in order to fully exploit its potentials and be appropriately handled.

\section{Teleconference as an educational medium and the educator's role}

According to Solomonidou (2006), the educator who is going to use a technologybased learning environment should be aware of and base the design of his/her 
teaching on learning theories that help in the development of a contemporary speculation regarding the constructive use of new technologies such as Constructivism (Scardamalia \& Bereiter, 2006). Furthermore, changing the class from conventional to technology-supported implies a change in the teaching model, where communication management is central to the educational role (Karakiza, 2010).

The (synchronous) teleconference as a form of interactive communication, assuming it is well-designed by the educator, has the potential to add value to the teaching / learning process, to enhance communication and interaction for the benefit of learners, while supporting the lead role and work of the educator (Milioritsas \& Georgiadi, 2010). With the use of teleconference a different role emerges for the educator, who is asked to use new flexible learning methods (Mouzakis, 2006; Milioritsas \& Georgiadi, 2010; Karakiza, 2010).

Teleconference can become an interactive educational tool, useful for the creation of flexible educational environments by means of DL. Its implemntation, in order to maximize the learning outcome, implies certain organizational conditions apart from the technological ones, i.e. the "operation" of the educator in an appropriate pedagogical framework so as to create conditions of student engagement (Anastasiades, 2007; Anastasiades, Manousou, Filippousis, Siakas, Koukoulis, Tomazinakis, Gkika, Mastoraki, Spanoudakis, Karagianni \& Karvunis, 2009). It is obvious that the use of teleconference requires educators to change their "lesson" plan, i.e. changes in their role, in order to meet its requirements (Valasidou, 2006).

From an educational perspective, the factors associated with the efficiency of the educational process through teleconference are linked with (Balaouras \& Mouzakis, 2008; Agorogianni, Zaharis \& Goudos, 2008; Kalogiannakis \& Vasilakis, 2008):

- The organization of content and its properly structured presentation.

- The preparation of educational material based on expected learning outcomes rather than the capabilities offered by technology.

- The presence of the educator, his movements and the way of conducting the course.

- The selection of teaching activities for the achievement of the expected learning outcomes, taking into account the technological problems that may arise.

- The implementation of teaching strategies that increase participant interaction.

- The shortening of the feeling of being at a distance.

- The position of technology, which should remain in the background.

Next paragraphs are presenting the positive and negative aspects derived by the usage of teleconferencing for supporting educational processes.

\subsection{Positive aspects}

The use of teleconference can enhance the interaction of the remote participants with the help of image, video and audio and facilitate communication between members of the virtual classroom. This attractive medium offers the opportunity to actively participate in real time and create collaborative learning conditions (Papadimitiou, Lampropoulou, Kampylis, Minaoglou, Vivitsou, Konetas, Paraskevas, Oikonomakos \& Gkikas, 2007). The "feeling of presence" alse, is also empowered (Anastasiades, 2008). Participants can expand their experiences, find new sources of information, receive alternative support depending on their learning style, interact in real time, 
observe, discuss and work together (Lampropoulou, Kampylis, Minaoglou, Papadimitiou, Vivitsou, Konetas, Oikonomakos, Gkikas, Paraskevas \& Grigoropoulos, 2008).

According to the Multiple Intelligence Theory of Gardner (1983), educators should take into account the multiple kinds of intelligence and learning styles of the students. The use of teleconference can help in this direction. Although the educator and the students either as a whole or individually may be located in different places, they have the ability to share information in an environment rich in resources and interact both with the educator and each other, building knowledge collaboratively.

Nowadays, the spread of broadband networks and the relatively inexpensive equipment makes it possible to transmit verbal and nonverbal messages via teleconference, approaching to some extent the type of face-to-face communication (Kotopouli, Mpasmatzidis, Koutli \& Kasidaki 2007). The findings of earlier researches (Bollom, Emerson, Fleming \& Williams, 1989; Angiolillo, Blonchard, Israelski \& Mane, 1997; Straus, Miles \& Levesque, 2001), according to which participants may not perceive and appropriately use information relative with the educator's gaze (they can't see the eyes of the speaker), lack of interaction, reluctance of learners to participate, low audio and video quality and lack of synchronization between audio and video signals, seem to fade away in contemporary reality, especially with high definition teleconference systems (Anastasiades, 2007).

\subsection{Negative aspects}

According to Martin (2005), the capabilities that technology and teleconference provide in today's DL are huge and its complete exploitation can drastically benefit learning communities, since among other things it can enhance the creation of collaboration conditions between participants. But, the lack of specific knowledge required by educators regarding teleconference's use, as well as its cost, are among the drawbacks that prevent teleconference from expanding and gaining implementation ground.

However and despite the aforementioned, teleconference represents a challenge for educators regarding the handling of the available technical equipment and the exploitation of the offered capabilities, in order to be more effective in their work.

\section{The technological aspect of teleconference}

There are many solutions to support distance learning with teleconference. Furthermore, there are many criteria to categorize these solutions. Examples of such criteria are the following: the number of learners; the cost of equipment; the type of client and or the access point (e.g. mobile device; teleconference room; web-page; personal computer, etc.); the type of hosting of the teleconference room (e.g. leasing of a teleconference room in an external provider; installation of a teleconferencing platform in the premises of the educational organization, etc.).

This section focuses on the specific decisions that an educational organization should undertake in order to reason about the required technological support of teleconferencing. These decisions are the following: 
- Teleconference scenario

One-to-many or many-to-many interaction? In case that we would like to support a virtual class with a big number of participating students (i.e. more than 50) then it would be a good idea to offer one (e.g. educator) to many (students) scenario where the feedback of the students is supported mainly by the text chat channel. In case that the educator would like to support a collaborative learning scenario (such as peerlearning, think-pair-share, etc.) then a many-to-many scenario should be supported among a rather small amount of participating students. In both cases it is important to take into account that one-to-many or many-to-many conferences may require technology that supports full screen, side-by-side screen, or cell-based screen layouts.

\section{- Teleconference place}

Teleconference room or desktop-based conference? In case that the teleconference is mainly offered in (and to) anyplace then it would be better to avoid the creation of a dedicated teleconference room and to choose desktop-based conference, which is more flexible and rather cheaper. The main criteria for that decision are (a) the location of the students (e.g. on-campus, off-campus, at regional locations, or at international locations) and (b) the need to set up ad-hoc videoconferencing sessions with the students on demand.

\section{- $\quad$ Offered functionality and compatibility with standards}

Proprietary or standard based? A key factor for the success of teleconferencing without creating more obstacles to the participants is to use a videoconferencing solution that is standards based. The well-known videoconferencing standards are ITU H.264 for video, G.711/G.722/G.729 for voice, and H.239/T.120 for data such as screen sharing or Web conferencing. Therefore for the basic functionality, the interoperability is possible. However if an organization would like to offer better quality and/or tele-presence the then interoperability is not a possibility. In case of better quality support, the videoconference platform should support, H.264 Scalable Video Coding (SVC) which is an emerging protocol. However, in such a case should be noted that interoperability among different H.264 SVC solutions isn't possible yet. For supporting telepresence, there are proprietary solutions such as Cisco's recently announced that it would release its Telepresence Interoperability Protocol (Cisco, n.d.).

\section{Methodology - sample}

In this paper we try to gain insight into the positive and negative aspects of teleconferencing for supporting educational processes. The methodology that has been deployed is based on semi-structured interviews of educators that have been used teleconference in education and there are teaching staff in tertiary education (e.g. Hellenic Open University) or vocational training staff big companies (e.g. Hellenic Telecommunications Organization).

More specifically, the sample of the conducted research presented in this paper has the following characteristics:

- Number of interviewees: sixteen (16) educators (14 male and 2 female)

- Age: 35-55 years old

- Prefecture of Achaia in Greece

- Education level: very high (all of them have bachelor and more than 55\% a $\mathrm{PhD}$ degree) 
It has to be noted that teleconference is not widely used in Greece as a technological medium in the learning process. Thus, it was extremely difficult to find and approach educators that could participate in our research.

The semi-structured interviews consisted of 12 questions (presented in section 5) and lasted from 30 to 55 minutes. All the questions were based on the literature review presented in the previous sections.

Each interview was recorded and when the data collection process was finished all the interviews were transcribed. Subsequently, common categories were created for each answer using content analysis (Bogdan \& Bilken, 1982; Mason, 2002). The content of this categorization is described in section 5.

\section{Findings and discussion}

This section presents the results of the semi-structured interviews, as well as some findings and observations inferred from the analysis and assessment of the given answers to the asked questions.

Q1. How many teleconferencing sessions for educational purposes have you conducted so far? What was the duration of each session?

The answers of the first question showed that the interviewees were rather experienced in teleconferencing use. More specifically, 4 of the interviewees $(25.0 \%)$ have conducted 2-5 teleconferencing sessions, 5 of them $(31.25 \%)$ have conducted 10 to 20 teleconferencing sessions and the remaining 7 (43.75\%) have conducted from 50 to 200 sessions. Each teleconferencing session lasted from 30 min to 3 hours.

Q2. Which were the subject of the teleconferencing sessions and the general characteristics of the trainees?

As it results from the sample's answers, the subjects of the sessions covered various topics, such as informatics, culture, marketing, etc., especially in tertiary education. The majority of the participants in the teleconferencing sessions were undergraduate and postgraduate students, educational institutions professors, employees and adults in general. If we focus on the answers provided in this question, based on the variety of objects and the differentiated characteristics of the participants, we could infer that the educators' answers are not polarized, meaning that they do not come from a single scientific domain.

Q3. Do you think that the educator should be well trained in the use of information and communication technologies in order to use teleconferencing as an educational tool?

Regardless of priorities, all the answers pointed out that the educators should be proficient in ICTs.

The majority of the interviewees (9 of them - 56.25\%) replied that the educators should be well trained in ICTs, in order to better exploit teleconferencing as an educational tool. 7 of them $(43.75 \%)$ said that under certain circumstances, such as the existence of high leveled technical support, a good training in ICTs is not necessary for the effective use of teleconference. Furthermore, they argued that the 
tools needed for the realization of a teleconferencing session are not difficult to use, requiring only some basic simple actions. However, they also agreed that knowledge of ICTs is necessary for the educators, in order to be aware of the modern developments and be able to fully exploit their features and capabilities in their work.

Q4. Do you think that the open and distance education is an important institution for the lifelong learning?

Totally, the sample has a generally positive attitude towards Open and Distance Learning (ODL), according to their answers in this question. Furthermore, 14 out of the 16 interviewees believe that ODL is truly an institution that comprises an important milestone in education. An indicative answer that represents this central belief is the following:

"Yes ODL is an important institution, because it facilitates the introduction of new objects in curriculums, while it attracts and engages large groups of learners that were excluded or marginalized due to specific social conditions (e.g. work requirements, place of residence, etc.)"

Two educators noted that teleconference is the most democratic institution in education, since it is available to everyone, regardless of economic, social, geographical, racial or other parameters.

On the other hand, there are two aspects, which without rejecting ODL they do not think of it as something so important in education and consider conventional education necessary.

Q5. Do you think that teleconferencing can support distance education, by enhancing the communication among learners and how can it do it?

In this question 14 interviewees $(87.5 \%)$ stated that teleconferencing could be a complementary tool in order to support flexible learning as well as distance education, by enhancing the interaction among learners. They believe that it fosters the possibility of dialogue and as long as the educator uses it appropriately, it can increase the quality of the interaction for the benefit of the learner.

Two of the interviewees (12.5\%) emphasized that teleconferencing cannot substitute face-to-face communication and face-to-face interaction but it can improve it in some cases.

Q6. What is the role of teleconferencing in the effective distance learning?

Concerning the effectiveness of teleconferencing from a learning perspective, the interviewees believe that there are many factors that could affect the effectiveness of a distance learning program. Examples are the learners' characteristics, the nature of the teaching subject, the educational objectives, the staff (academic, technical assistant) engaged in this program, the teaching methodology, the technological equipment of teleconferencing rooms, etc. Everyone agreed that teleconferencing plays a complementary role in ODL. They consider it as an important tool, which in combination with other approaches enhances communication and learning. An indicative answer for the preceding is the following:

"Teleconferencing is a cornerstone in ODL, since it is the direct means of communication and interaction between the learner and the educator". 
Q7. Do you think that teleconferencing can enhance collaborative learning, and if so to what extent?

The majority of the educators (12 of them, 75.0\%), answering this question, believe that teleconferencing augments collaborative learning to an extended degree because it allows the participants to work in groups, exposing multiple views and learning together. However, there is one other view, according to which the collaborative learning's support depends on the frequency of conduction, the participants' characteristics and, mainly, on the educators' expertise.

Q8. What are the difficulties faced by the educator in the deployment of teleconferencing in order to support distance education? What are your suggestions for eliminating or reducing these difficulties?

Regarding the difficulties and the obstacles of teleconferencing use in distance education, all the educators agreed that the difficulties encountered in the deployment of teleconferencing mainly have a technical nature. More specifically, the main problem is the lack of expertise, while a secondary one is the limited access to technological equipment.

An additional view focused on the heterogeneity of the learners and their coordination.

To avoid these problems the educators mainly suggested infrastructures that provide sufficient bandwidth and good planning in terms of time of the processes to be carried out in a teleconference. They gave low priority to the pedagogical aspect, although it appears that some of them know that pedagogical knowledge is necessary to conduct an effective virtual meeting through teleconference.

Q9. What characteristics could both learners and educators identify in the deployment of teleconferencing as a supplementary learning tool in distance education?

According to the feedback received for this question, the educators believe that teleconferencing could literally be an effective tool for them because of its unique affordances. More specifically, five of them (31.25\%) claimed that teleconferencing propounds the strength of computer systems, which facilitate easy access and sharing of educational content for educators and students.

Five of them $(31.25 \%)$, noted that the role of the educator changes while using teleconference. The educator becomes more coordinative in a student-centered system of teaching / learning, which offers a large degree of freedom to the participants and independence of space and time while conducting learning sessions. In combination with the traditional teaching methods, teleconference creates a hybrid learning model, through which the collaborative learning can be supported when appropriate interactive material is used.

Four of them $(25.0 \%)$ responded that teleconferencing can substitute the lack of physical presence to some extent and reduce the distance between team members. It can also play an important role to increase the interaction between educator and learners, as well as among the learners.

Two of them $(12.50 \%)$, replied that teleconferencing may cover some gaps of ODL, provided that the learners will receive guidance in advance. Judging by their answers, they seem to recognize the possibility of creating diverse problems to 
students, either relative with the infrastructure or with their ability to use technologies.

Q10. In your opinion, what situation is stressful for the educators who are conducting teleconference to assist the learning process?

The whole sample suggested that the educators should be well prepared before the tele-lecture, as well as prepare and guide the learners in order to conduct a smooth teleconferencing session.

The most stressing issue for the educators refers to whether the teleconference system will provide the desired functionality in order to constitute an effective learning medium. The following answer is characteristic, inclusive and, as far as we are concerned, representative of the overall interviewees' perspectives:

"Beyond the aforementioned technical difficulties that put stress mainly upon those with limited or no knowledge on new technologies, begetting fear of an improper lesson conduct, the instructor faces the difficulty of non-direct contact with learners. Although there is usually visual contact (indeed many systems place emphasis on providing high definition video) and interactive audio exchange, the contact is indirect and the non-verbal indications are often vague and unspecified, as the available information is limited to what the medium is able to display. Therefore, the instructor cannot fully assess the "messages" of visual contact and gaze. For this reason, the educator has to realize and compromise with the fact that while most teleconference media dispose functions and structures that seem to be identical to a physical classroom, the learning process is different from the traditional one."

One other reply states:

"Two issues provoke stress: the use and performance of the teleconferencing tool and the fact that the educator is not "seeing" and interacting with the learners using all of his/her senses."

As shown, the educators appear to be "technophobic" not so much regarding the use of teleconferencing, as for the result of its use. This happens due to the fact that through teleconferencing, teaching enters in new dimensions and the need of methodical training emerges in order for the trainers to be able to respond to upcoming challenges.

Q11. In your opinion, what are the major advantages of teleconferencing use as an educational tool, from the instructors' point of view?

Ten of the interviewees $(62.5 \%)$ answered that they perceive teleconference as a "helpful assistant" in the educational process. One of them even considered teleconference as "one-way" in distance education.

Furthermore from all answers the following advantages of the teleconferencing use are detected:

- Time saving.

- Reduced transportations.

- Expansion of educators' knowledge in new technologies.

- Ability to record the activity in a teleconference session so that the student can rewatch it at will.

- Creation of added value in distance learning.

- Provision of teaching and social interaction potentials between student groups. 
- Participation in a lesson regardless of the educator's or the student's location

- Creation of an environment for direct contact.

- Increase of the capabilities for more direct and frequent communication.

Q12. In your opinion, what are the main disadvantages of teleconferencing use as an educational tool, from the instructors' point of view?

From the whole sample the following disadvantages of the teleconferencing use are detected:

- The unfamiliarity of the participants with the technology.

- In a teleconference session, educators should have a certain attitude towards the camera and stay close to microphones; thus, they should quickly familiarize themselves with these requirements to avoid losing valuable time. Every "old" teaching technique should be adapted to the new teaching potentials and limitations.

- The stress of users to be disconnected due to problematic network connection.

- The uncertainty of the participants about the effectiveness of the teleconferencing in education.

- The lack of face to face communication.

- The coordination in relation to the lack of time for the development of questions from students.

- The absence of the non-verbal or of the body messages.

- Educators' limited knowledge about educational methods and techniques for distance education and adult education.

- The lack of technical support.

- The rather weak active participation of the learners.

- The big amount of time required to prepare a teleconference lesson.

- Technological issues such as:

$\checkmark$ Low internet speed.

$\checkmark$ Low quality infrastructure.

$\checkmark$ Cost of Internet connection and purchase modern computer.

$\checkmark$ Lack of staff for technical support.

$\checkmark$ The quality of the communication that depends on the quality of the underlying platform.

\section{Conclusion and Future Work}

This paper focused on assessing the usage of teleconference to support distance learning by questioning a group of educators over several teleconference-related issues. After a quick literature review concerning the role of teleconference as an educational medium and the educator's role in an educational process that involves teleconferencing, many pros and cons of teleconferencing in education have been recorded. These pros and cons were considered as a reference point for our research both in terms of validation and questions formulation.

Our research was conducted in the Prefecture of Achaia, Greece, where we collected the opinions of sixteen (16) educators through semi-structured interviews and after the analysis of their feedback we were led to several outcomes. This study could conclude that teleconference can be an effective medium for conducting ODL lessons offering a learning environment that quashes distance limitations and increases interaction between educator and learners as well as among the learners. 
Furthermore, the ability of participants to communicate with each other while remaining at their own physical spaces provides strong motivation for active engagement in the learning process and creates enthusiasm among learners.

Besides these fundamental aspects, teleconference offers several secondary, yet significant, benefits when used in education. For example, it helps participants to develop technological skills, perform both formal and informal educational meetings as frequent as they want, improve their social interactions and create a digital record of conducted lessons for review purposes.

On the other hand, the negative aspects of using teleconference in education are tightly connected with technical difficulties that affect its effectiveness in several ways. For instance, such difficulties may have a negative impact in the educator's preparation for the online class, or result in a low quality communication between a session's participants that negatively affects the learning process.

Finally, considering the overall evaluation results it turns out that the positive aspects outmatch the negative ones from a quality perspective. In general, the educators converge that teleconference should meet a wider implementation in distance learning, under some certain conditions.

Our future research plans will focus on the students' opinions about teleconference in education. The objective is to highlight the major parameters that affect the interaction between educator-students and students-students in a teleconference mediated teaching environment from the students' perspective.

\section{References}

Agorogianni, A., Zaharis, Z. \& Goudos, S. (2008). Synchronous tele-education: The introduction of new technologies in the educational process and case studies of their exploitation in the Aristotle University of Thessaloniki. In P. Anastasiades (ed.) Teleconference in the service of Lifelong and Distance Learning (pp. 133-179). Athens: Gutenberg.

Anastasiades, P. (2006). Interactive Videoconferencing in K-9 Education: "ODUSSEAS 2000-2004" a case study in Elementary Schools in Greece and Cyprus. Proceedings of the Diverse 2006, 6th International Conference on Video and Video-conferencing in Education.5-7 July, 2006, Scotland, Glasgow: CaledonianUniversity.

Anastasiades, P. (2007). The educational use of interactive teleconference in the modern school: Socioconstructivist approach. In A. Lionarakis (ed.) Proceedings of $4^{\text {th }}$ International Conference in Open and Distance Learning - Forms of Democracy in Education: Open Access and Distance Education, Section B, pp.668-681.

Anastasiades, P. (2008). Issues of pedagogical planning for educational use of teleconference in an environment of mixed-polymorph-learning. InP. Anastasiades (ed.), Teleconference in the service of Lifelong and Distance Learning (pp. 17-89). Athens: Gutenberg.

Anastasiades, P., Manousou, E., Filippousis, G., Siakas, S., Koukoulis, N., Tomazinakis, A., Gkika, P., Mastoraki, E., Spanoudakis, A., Karagianni, D. \& Karvunis, L. (2009). Teleconference in the service of collaborative construction of knowledge and interdisciplinary approach. From Theory to Practice: «ODUSSEAS 2009: Environment - Mediterranean Sea - Renewable Energy Sources. In A. Lionarakis (ed.) Proceedings of $5^{\text {th }}$ International Conference in Open and Distance Learning, Section A, pp. 111-124.

Anastasiades, P., Filippousis, G., Karvunis, L., Siakas, S., Tomazinakis, A., Giza, P. \& Mastoraki, H. (2010).Interactive Videoconferencing for collaborative learning at a distance in the school of 21st century: A case study in elementary schools in Greece. Computers and Education, 54, 2, pp. 321-339. 
Angiolillo, J.S., Blonchard, H., E., Israelski, E.W. and Mane, A. (1997). Technology constrains of video mediated communication Ink E Finn, A. J. Sellen \& S. B. Wilbur (eds), Video mediated communication (pp. 51-74). Mahwah NJ: Lawrence Erbaum Associates.

Balaouras, P. \& Mouzakis, C. (2008). Design, implementation and evaluation of an inter-institutional training course of technicians in issues relative with teleconference services. In P. Anastasiades (ed.), Teleconference in the service of Lifelong and Distance Learning (pp. 93132). Athens: Gutenberg.

Beldarrain, Y. (2006). DistanceEducationTrends: Integrating newtechnologiesto foster student interaction and collaboration. Distance Education, 27, 2, pp. 139-153.

Baker, S., Wentz, R. \& Woods, M. (2009).UsingVirtualWorlds in Education: Second Life ${ }^{\circledR}$ as an Educational Tool. Teaching of Psychology, 36, 1, pp. 59-64.

Bogdan, R., Bilken, S. (1982). Qualitative Research for Education: An Introduction to Theory and Methods. Boston: Allyn \& Bakon inc.

Bollom, C. E., Emerson P. A., Fleming, P. R. and Williams A. R. (1989).The Charing Cross and Westminister Interactive television network. Journal of education Television, 15, 1, pp. 5-15.

Cisco (n.d.). Cisco TelePresence and the Telepresence Interoperability Protocol. Retrieved 22/2/2013 from http://www.cisco.com/en/US/prod/collateral/ ps7060/ps8329/ps8331/ps7315/q_a_c67580285.html

Edirisingha, P., Nie, M., Pluciennik, M. \& Young, R. (2009). Socialisation for learning at a distance in a 3-Dmulti-user virtual environment. British Journal of Educational Technology, 40, 3, pp. 458-479.

Ellaway, R., Dewhurst, D. \& Cumming, A. (2003). Managing and supporting medical education with a virtual learning environment: The Edinburgh Electronic Medical Curriculum. MedTeach, 25, 4, pp. 372-380.

Hatzipanagos, S. \& Warburton, S. (2009). Feedback as dialogue: exploring the links between formative assessment and social software in distance learning. Learning, Media and Technology, 34, 1, pp. $45-59$.

Hoffman, E. (2009). Evaluating social networking tools for distance learning. Worldwide Online Conference in Technology, Colleges and Community (TCC), Honolulu, HI, 14-16 April 2009, pp. 92-100.

Johnson, C. M., Corazzini, K. N., and Shaw, R. (2011). Assessing the Feasibility of Using Virtual Environments in Distance Education. International Journal in Knowledge Management \& ELearning, 3, 1, pp. 5-16.

Kalogiannakis, M. \& Vasilakis, K. (2008). Empirical approaches of teleconference's application. The case of the University of Crete. In P. Anastasiades (ed.), Teleconference in the service of Lifelong and Distance Learning (pp. 379-426). Athens: Gutenberg.

Karakiza, T. (2010). Looking at the screen, touching the keyboard keys. Conversions of pedagogical relationship in a networked environment. Athens: Papazisis Press.

Kotopouli, G., Mpasmatzidis, G., Koutli, M. \&Kasidaki, S. (2007). The role of teleconference in Distance Learning. Athens: department of Primary Education, University of Athens. Retrieved 22/10/2012 from http://pse.primedu.uoa.gr/foitites/garifalia_kotopouli/Ergasies/anastasiades.pdf

Lampropoulou, N., Kampylis, P., Minaoglou, N., Papadimitiou, S., Vivitsou, M., Konetas, D., Oikonomakos, E., Gkikas, A., Paraskevas, M. \& Grigoropoulos, E. (2008). Teleconference units and their didactic exploitation in online learning. In P. Anastasiades (ed.), Teleconference in the service of Lifelong and Distance Learning (pp. 225-272). Athens: Gutenberg.

Martin, M. (2005). Seeing is believing: the role of videoconferencing in distance learning. British Journal of Educational Technology, Vol. 36, No 3, pp. 397-405.

Mason, J. (2002). Qualitative Researching, $2^{\text {nd }}$ Edition. London: Sage Publications.

McBrien, J. L., Cheng, R. \& Jones, P. (2009). Virtual Spaces: Employing a Synchronous Online Classroom to Facilitate Student Engagement in Online Learning. The International Review of Research in Open and Distance Learning, 10, 3. Retrieved 12 October 2012 from http://www.irrodl.org/index.php/irrodl/article/view/605/1264

Milioritsas, E. \& Georgiadi, E. (2010). Teleconference's impact in the learning process of the Hellenic Open University - Views of students and Tutors of Thematic Modules EKP65 and EKE50. Open Education- The Journal for Open and Distance Educational Technology, 6, 1 \& 2, 152 - 
157.

Mouzakis, C. (2006). Information and Communication Technologies in Adult Education. Athens: Institute of Continuous Adult Education (IDEKE).

Panagiotakopoulos, C., Lionarakis, A., Xenos, M. (2003). Open and Distance Learning: Tools of Information and Communication Technologies for Effective Learning. Proceedings of the Sixth Hellenic-European Conference on Computer Mathematics and its Applications, HERCMA 2003. Athens 25-27/9/2003, Greece, pp. 361-367.

Papadimitiou, S., Lampropoulou, N., Kampylis, P., Minaoglou, N., Vivitsou, M., Konetas, D., Paraskevas, M., Oikonomakos, E., Gkikas, A. (2007). The Multiple Use of Teleconference in Distance Learning of Greek Educators through the Hellenic School Network. (ed.) Proceedings of $4^{\text {th }}$ International Conference in Open and Distance Learning - Forms of Democracy in Education: Open Access and Distance Education, Section A, pp. 521-530.

Perraton, H. (1988). A theory for distance education. In Distance education: International perspectives, ed. D. Sewart, D. Keegan, and B. Holmberg (pp. 34-45). New York: Routledge.

Roberts, R. (2009). Video conferencing in distance learning: A New Zealand schools' perspective. Journal of Open, Flexible and Distance Learning, 13, 1, pp. 91-107.

Rozi, E. (2007). A study of protocols and tools for teleconference. Master Thesis. Patra: University of Patras, Polytechnic School, Department of Computer Engineering and Informatics.

Simpson, O. (2002). Supporting Students in open and Distance Learning. London: KoganPage.

Solomonidou, C. (2006). New trends in educational technology. Constructivism and contemporary learning environments. Athens: Metaihmio.

Trueb, G., Lammers, S. \&Calyam, P. (2007). High Definition Videoconferencing: Codec Performance, Security, and Collaboration Tools. Retrieved 5/10/2012 from http://www.adec.edu/nsf/reureport-2007-osu.pdf

Tzanakos, N. (2012). The positive and negative aspects in the use of teleconference as an educational tool, from the perspective of educators. Master Thesis. Patra: Hellenic Open University.

Valasidou, A. (2006). Guide of methods for Distance Learning. Retrieved 12/12/2011 from http://195.251.22.132/tele edu/Uploads/distanceteachingmethods.pdf

Straus, S. G., Miles, J. A., Levesque, L. L. (2001). The effects of videoconference, telephone, and faceto-face media on interviewer and applicant judgments in employment interviews. Journal of Management 27(2001), pp. 363-381. 